Pacific Journal of Mathematics

SMASH PRODUCTS, INNER ACTIONS AND QUOTIENT RINGS 


\title{
SMASH PRODUCTS, INNER ACTIONS AND QUOTIENT RINGS
}

\author{
Miriam COHEN
}

\begin{abstract}
Let $H$ be a Hopf algebra over a field $k$, and $A$ an $H$-module algebra over $k$. Let $A^{H}=\{a \in A \mid h \cdot a=\varepsilon(h) a$, all $h \in H\}$. This paper is mainly concerned with inner actions. We prove the existence of a "symmetric" quotient ring $Q$ of $A$, which is also an $H$-module algebra, and consider $Q$-inner actions, an analogue of $X$-inner automorphisms. Under certain conditions on $A$ and $H$ we show that $Q$ contains $B$, a finite-dimensional separable algebra over its center $C$, a field. Moreover, the centralizer of $B$ in $Q$ is $Q^{H}$. This is used to prove that if $A^{H}$ is P.I. then so is $A$, and that $A$ is fully integral over $A^{H} C$ of bounded degree. We also consider connections between the $A, A^{H}$ and $A \# H$ module structures.
\end{abstract}

0. Introduction. Let $H$ be a Hopf algebra over a field $k$. Let $A$ be an $H$-module algebra over $k$. As in [6], let $A \# H$ denote the so called smash product and

$$
A^{H}=\{a \in A \mid h \cdot a=\varepsilon(h) a, \text { all } h \in H\} .
$$

That is, $A^{H}$ is the set of elements of $A$ on which the action of $H$ is trivial. This paper is mainly concerned with inner actions of $H$ on $A[4,15]$.

In $\$ 1$ we prove some basic identities (Lemma 1) which enable us, among the rest, to prove the existence of left and "symmetric" quotient rings $Q$ (with respect to $\mathscr{F}=\{I \mid I$ is an $H$-stable ideal of $A$ with $\left.r_{A}(I)=l_{A}(I)=0\right\}$ ) which are also $H$-module algebras. (Right quotient rings were introduced in [7]). These quotient rings give rise to a more generalized notion of inner actions, an example of which is that of $X$-inner automorphisms [13].

Another consequence of Lemma 1, which is not in the mainstream of this paper, is Proposition 4 which states that if $H$ is cocommutative then the center of $A$ is $H$-stable.

In $\$ 2$ we get to the main concern of this paper, that of inner actions. In [4] we have shown that inner actions give rise to 2-cocycles $t$, so that $A \# H \cong A_{t}[H]$, a twisted product. In $A_{t}[H], 1 \otimes H$ commutes with $A$, a key property utilized in $\S 3$. In Proposition 8 and Lemma 9 we summarize basic facts about $A$ and $A_{t}[H]$. We proceed to consider "generalized" inner actions. That is, inner actions induced by elements in the right or 
left quotient rings $\left(Q^{r}\right.$ or $\left.Q^{l}\right)$. In Proposition 12 we show that in some sense these inducing elements are "normalizing". More precisely we show that if the action is $Q^{r}$ or $Q^{l}$-inner then it is induced by elements in the smaller, symmetric rings of quotients, $Q^{s}$; hence satisfy a property known for generalized inner automorphisms or derivations. Namely: for each $h \in H$, there exists an ideal $I \in \mathscr{F}$ so that $u(h) I, \nu(h) I, I u(h)$ and $I \nu(h)$ are all contained in $A$. Specifying to $Q$-inner actions on $H$-prime algebras we show in Proposition 14 that $Q$ must then be prime and centrally closed, with center $C$, the extended centroid of $Q$. If $H$ is furthermore assumed to be $n$-dimensional and semisimple we show in the course of proving Proposition 16 that $C_{t}[H] \subset Q_{t}[H]$ is a separable $n$-dimensional $C$-algebra. This fact is used to prove that if $H$ and $A$ satisfy all the above conditions then $Q$ contains $B$, a separable $C$-algebra of dimension $\leq n$.

The algebra $B$ is the analogue of the "algebra of the group", a known algebra in the study of groups acting as automorphisms. It "mirrors" $H$ in $A$. Moreover, $C_{T}(B)$, the centralizer of $B$ in any $T \subset Q$ satisfies:

$$
C_{T}(B)=T^{H} .
$$

We are thus in a position to apply a theorem of Montgomery and Smith [12] about centralizers of separable subalgebras. We show in Theorem 17 that if $H$ is an $n$-dimensional semisimple Hopf algebra acting in an inner fashion on $A$. Then, if $A^{H}$ satisfies a P.I. so does $A$.

Another application of Proposition 16 is to a question about integrality. An application of a theorem of Passman [14] yields Theorem 18: If $A$, $H$ are as in Theorem 17 and $A$ is moreover assumed to be $H$-prime then $A$ is fully integral over $A^{H} C$ of bounded degree $t$.

In $\S 3$ we consider $A$-modules when the action of $H$ on $A$ is inner and $H$ is $n$-dimensional and semisimple. Let $V_{A}$ be a right $A$-module, then $V$ is an $A^{H}$-module by restriction of "scalars", moreover $V_{A}$ induces an $A_{t}[H]$ module $\bar{V}=V \otimes_{A} A_{t}[H]$. Using a Maschke-type theorem for Hopf algebras [6], we prove Theorem 21 which relates the module structures of $V_{A}, V_{A^{H}}$ and $\bar{V}_{A_{t}[H]}$. This is done in an analogous manner to Passman and Lorenz's treatment of the case $H=k G, G$ a finite group of automorphism of $A$ [10]. We further consider $A_{t}[H], A$ and $A^{H}$-bimodules, using a two-sided Maschke theorem proved in $§ 1$. Finally, we apply these results to central simple algebras. In Theorem 23 we prove that if $H$ is assumed to be cocommutative and pointed, in addition to being $n$-dimensional and semisimple, and $A$ is central simple then $A$ is fully integral and finitely generated over $A^{H}$, and $A^{H}$ is a finite direct sum of simple rings. In 
particular, if $H=u(L)$, the universal enveloping algebra of an $n$-dimensional semisimple restricted Lie algebra $L$ acting as derivation on $A$, then $A^{L}$ is a finite direct sum of simple rings (Corollary 24).

1. Preliminaries. Let $H$ be a Hopf algebra over a field $k$ and $A$ an $H$-module algebra. Notation is as in [19], for a summary of basic properties see [6]. Let $S$ denote the antipode of $H, \Delta$ the comultiplication map, $\varepsilon$ the augmentation map. Denote for each $h \in H$

$$
\Delta(h)=\sum_{(h)} h_{1} \otimes h_{2} .
$$

We start with a basic identity:

Lemma 1. Let $H$ be a Hopf algebra and $A$ an $H$-module algebra. For any $a, b \in A, h \in H$

$$
(h \cdot a) b=\sum_{(h)} h_{1} \cdot\left[a\left(S\left(h_{2}\right) \cdot b\right)\right] .
$$

If $S$ is bijective then

$$
a(h \cdot b)=\sum_{(h)} h_{2} \cdot\left[\left(S^{-1}\left(h_{1}\right) \cdot a\right) b\right] .
$$

Proof. (1)

$$
\begin{aligned}
\sum_{(h)} h_{1} \cdot\left[a\left(S\left(h_{2}\right) \cdot b\right)\right] & =\sum_{(h)}\left(h_{1} \cdot a\right)\left(h_{2} \cdot S\left(h_{3}\right) \cdot b\right) \\
= & \sum_{(h)}\left(h_{1} \cdot a\right)\left(\varepsilon\left(h_{2}\right) b\right)=\left[\sum_{(h)} h_{1} \varepsilon\left(h_{2}\right)\right] b=(h \cdot a) b
\end{aligned}
$$

(2) is proved similarly.

This lemma has some immediate corollaries.

COROllary 2. If $I$ is an $H$-stable subring of $A$ then the left annihilator, $l_{A}(I)$, is $H$-stable. Moreover, if $S$ is bijective then the same is true for the right annihilator of $I$.

Proof. Let $b \in I, a \in l_{A}(I)$, then by Lemma 1

$$
(h \cdot a) b=\sum_{(h)} h_{1} \cdot\left[a\left(S\left(h_{2}\right) \cdot b\right)\right]=\sum_{(h)} h_{1} \cdot 0=0 .
$$

Hence $h \cdot a \in l_{A}(I)$ and thus $l_{A}(I)$ is $H$-stable. One uses part (2) of Lemma (2) to prove the statement for $r_{A}(I)$. 
An $H$-module algebra is called $H$-prime (respectively $H$-semiprime) if for any $H$-stable ideals $I, J$ of $A$, then $I J=0$ (resp. $I^{2}=0$ ) implies $I=0$ or $J=0$ (resp. $I=0$ ).

Using Corollary 2 and the usual proof for semiprime rings it is immediate that:

Corollary 3. Let $H$ be a Hopf algebra, and $A$ an $H$-semiprime algebra. If $I \neq 0$ is an $H$-stable ideal of $A$, then

$$
r_{A}(I)=l_{A}(I) \text { and } I \cap r_{A}(I)=0 .
$$

If moreover $A$ is $H$-prime then $r_{A}(I)=l_{A}(I)=0$.

This result will be useful shortly when we form quotient rings.

Another interesting consequence of Lemma 1 is the following:

Proposition 4. Let $H$ be a cocommutative Hopf algebra and $A$ an $H$-module algebra. Then, $Z(A)$, the center of $A$, is $H$-stable.

Proof. First let us note that $S$ is bijective since $S^{2}=\mathrm{id}$, and thus $S=S^{-1}[19]$. Now say $z \in Z(A), a \in A$, then,

$$
\begin{aligned}
(h \cdot z) a & =\sum_{(h)} h_{1} \cdot\left[z\left(S\left(h_{2}\right) \cdot a\right)\right] \quad(\text { Lemma } 1) \\
& =\sum_{(h)} h_{1} \cdot\left[\left(S\left(h_{2}\right) \cdot a\right) z\right] \quad(z \in Z(A)) \\
& =\sum_{(h)} h_{1} \cdot\left[\left(S^{-1}\left(h_{2}\right) \cdot a\right) z\right] \quad\left(S=S^{-1}\right) \\
& =\sum_{(h)} h_{2} \cdot\left[\left(S^{-1}\left(h_{1}\right) \cdot a\right) z\right] \quad(H \text { is cocommutative }) \\
& =a(h \cdot z) \quad(\text { Lemma } 1)
\end{aligned}
$$

The above proof is R. J. Blattner's "cleaner" version of our original argument, we thank him for pointing it out to us. The assumption about the cocommutativity of $H$ cannot be removed, for, as shown in [2], there exist graded rings (i.e. $H=(k G)^{*}, G$ a non-abelian group) whose center is not graded.

We turn now to quotient rings. In [7] we have introduced a right quotient ring $Q^{r}$ with respect to:

$$
\mathscr{F}=\left\{I \mid I \text { is an } H \text {-stable idea of } A \text {, with } r_{A}(I)=l_{A}(I)=0\right\} \text {. }
$$

Note that by Corollary 3 , if $A$ is $H$-semiprime then $\mathscr{F}=\left\{I \oplus \operatorname{ann}_{A} I \mid\right.$ $I$ is an $H$-stable ideal of $A\}$. If $A$ is $H$-prime, then $\mathscr{F}=\{I \mid 0 \neq I$ is an $H$-stable ideal of $A\}$. 
Let us recall how $Q^{r}$ became an $H$-module algebra. Let $q \in Q^{r}$, where $q: I_{A} \rightarrow A$, then for any $h \in H, h \cdot q: I_{A} \rightarrow A$ is defined by:

$$
(h \cdot q)(y)=\sum_{(h)} h_{1} \cdot\left[q\left(S\left(h_{2}\right) \cdot y\right)\right], \quad \text { all } y \in I .
$$

If $H$ has a bijective antipode, we can define analogously an action of $H$ on $Q^{l}$. Where each element $q=[f] \in Q^{l}$ is an equivalence class of left $A$-module homomorphisms, $f:{ }_{A} I \rightarrow A$, where $I \in \mathscr{F}$ (F्F as above). As usual $f$ is written to the right of the element on which it acts and we identify $q$ with $f$. Define then for any $h \in H, f$ as above; $h \cdot f:{ }_{A} I \rightarrow A$ by:

$$
x(h \cdot f)=\sum_{(h)} h_{2} \cdot\left[S^{-1}\left(h_{1}\right) \cdot x\right], \quad \text { all } x \in I .
$$

Using known identities for $S^{-1}[6,19]$ one can proceed as in the proof for $Q^{r}$ to show:

Proposition 5. Let $H$ be a Hopf algebra with bijective antipode, $A$ an $H$-module algebra. Let $Q^{l}$ be the left quotient ring of $A$ with respect to $\mathscr{F}$, then under the above action, $Q^{l}$ is an $H$-module algebra. This action extends the action of $H$ on $A$.

In many instances either of these quotient rings is too large. Considering $X$-inner automorphism, it turned out to be sufficient to deal with the normal closure of a ring [13]. Similarly, for $Q$-inner derivations [9] one only needed to deal with a certain two-sided quotient ring. Both can be viewed either as the intersection of the left and right quotient rings, or as limits of "multiplier algebras" that occur in the study of "partly inner" automorphisms of $C^{*}$-algebras $[16,17]$.

In [15], Passman termed such quotient rings, "symmetric", and studied them for certain prime rings (where $\mathscr{F}=\{$ all non-zero ideals of $R\}$ ).

As in [15], let $Q^{s}$ denote the symmetric ring of quotients with respect to $\mathscr{F}$. It consists of equivalence classes of pairs $(f, g)$ where $f \in Q^{l}$ and $g \in Q^{r}, f:{ }_{A} I \rightarrow A, g: J_{A} \rightarrow A$ satisfying the associativity condition:

$$
(x f) y=x(g y), \quad \text { all } x \in I, y \in J \text {. }
$$

We extend the action of $H$ to $Q^{s}$ in the obvious manner. That is, for $s=(\overline{f, g})$ as above, $h \in H$, define:

$$
h \cdot s=(\overline{h \cdot f, h \cdot g}) .
$$

Proposition 6. Let $H, A, \mathscr{F}$ be as in Proposition 6. Let $Q^{s}$ be the symmetric quotient ring with respect to $\mathscr{F}$, then the above action makes $Q^{s}$ an $H$-module algebra and extends the action of $H$ on $A$. 
Proof. All we must check is that the pair $(h \cdot f, h \cdot g)$ satisfies the above associativity condition. Let $x \in I, y \in J$ then

$$
\begin{aligned}
{[x} & (h \cdot f)] y=\sum_{(h)}\left[h_{2} \cdot\left[\left(S^{-1}\left(h_{1}\right) \cdot x\right) f\right]\right] y \\
= & \sum_{(h)} h_{2} \cdot\left[\left[\left(S^{-1}\left(h_{1}\right) \cdot x\right) f\right]\left(S\left(h_{3}\right) \cdot y\right)\right] \quad(\text { Lemma 1.1) } \\
= & \sum_{(h)} h_{2} \cdot\left[\left(S^{-1}\left(h_{1}\right) \cdot x\right)\left[g\left(S\left(h_{3}\right) \cdot y\right)\right]\right] \quad((f, g) \text { satisfies associativity) } \\
= & \sum_{(h)}\left[h_{2} S^{-1}\left(h_{1}\right) \cdot x\right]\left[h_{3} \cdot\left(g\left(S\left(h_{4}\right) \cdot y\right)\right)\right] \\
= & \sum_{(h)}\left(\varepsilon\left(h_{1}\right) x\right)\left[h_{2} \cdot\left(g\left(S\left(h_{3}\right) \cdot y\right)\right)\right] \\
= & x \sum_{(h)} h_{1} \cdot\left(g\left(S\left(h_{2}\right) \cdot y\right)\right)=x[(h \cdot g) y] .
\end{aligned}
$$

We have shown that, $[x(h \cdot f)] y=x[(h \cdot g) y]$, hence that $(h \cdot f$, $h \cdot g$ ) satisfies the associativity condition. As in [15], an alternate way of viewing $Q^{s}$ is:

$$
\begin{aligned}
Q^{s} & =\left\{q \in Q^{r} \mid I q \subset A \text { and } q I \subset A, \text { some } I \in \mathscr{F}\right\} \\
& =\left\{q \in Q^{l} \mid I q \subset A \text { and } q I \subset A, \text { some } I \in \mathscr{F}\right\} .
\end{aligned}
$$

Well, in order to show that $H$ acts on $Q^{s} \subset Q^{r}$ we must show it is $H$-stable. Let $q \in Q^{s}$, and $I \in \mathscr{F}$ so that $I q \subset A$ and $q I \subset A$. Let $h \in H$, $a \in I$, then: $a(h \cdot q)=\sum_{(h)} h_{2} \cdot\left[\left(S^{-1}\left(h_{1}\right) \cdot a\right) q\right]$ (by Lemma 1$)$. Since $I$ is $H$-stable, and since $I q \subset A$, this expression is contained in $H \cdot A \subset A$. Hence $I(h \cdot q) \subset A$. By definition, $(h \cdot q) I \subset A$.

Recall that when $H$ is a finite-dimensional Hopf algebra then $S^{-1}$ exists. If it is also semisimple, then $H$ is a separable algebra over $k$, and it contains an idempotent $e$ satisfying:

$$
e h=h e=\varepsilon(h) e, \quad \text { all } h \in H .
$$

$e$ is an element in the so called integral of $H$.

Finally, as for group actions [13], let us give a "right", and "two-sided" Maschke-type theorem, analogous to the "left"-version proved in [6].

Proposition 7. Let $H$ be a finite-dimensional semisimple Hopf algebra. Let $A$ be an $H$-module algebra.

(1) If $V_{A \# H}$ is a right $A \# H$ module and $W_{A \# H}$ is an $A \# H$ submodule of $V$ which is an $A$-module direct summand of $V$, then it is an $A \# H$-direct summand of $V$. 
(2) If $V$ is an ( $A \# H, A \# H)$-bimodule, and $W$ is an ( $A \# H, A \# H)$-subbimodule of $V$ which is an $(A, A)$-direct summand of $V$ then $W$ is an $(A \# H, A \# H)$-direct summand of $V$.

Proof. (1) The proof is similar to [6]. Let $\lambda: V \rightarrow W$ be the $A$-module projection. Let $e$ be the idempotent in the integral, for each $\nu \in V$ define:

$$
\tilde{\lambda}(\nu)=\sum_{(e)} \lambda\left(\nu \cdot e_{2}\right) \cdot S^{-1}\left(e_{1}\right) .
$$

We show that $\tilde{\lambda}$ is an $A \# H$-module map. Well, if $a \in A$ then

$$
\tilde{\lambda}(\nu) \cdot a=\sum_{(e)} \lambda\left(\nu \cdot e_{2}\right) \cdot S^{-1}\left(e_{1}\right) a,
$$

however

$$
h a=\sum_{(h)}\left(h_{1} \cdot a\right) h_{2}, \quad \text { all } h \in H .
$$

Thus the above equals $\sum_{(e)} \lambda\left(\nu \cdot e_{3}\right) \cdot\left[S^{-1}\left(e_{2}\right) \cdot a\right] S^{-1}\left(e_{1}\right)$. Since $\lambda$ is right $A$-module map this is

$$
\begin{aligned}
\sum_{(e)} \lambda\left(\nu \cdot \left[e_{3}\right.\right. & \left.\left(S^{-1}\left(e_{2}\right) \cdot a\right)\right] \cdot S^{-1}\left(e_{1}\right) \\
& =\sum_{(e)} \lambda\left(\nu \cdot\left[\left(e_{3} S^{-1}\left(e_{2}\right) \cdot a\right) e_{4}\right]\right) \cdot S^{-1}\left(e_{1}\right) \\
& =\sum_{(e)} \lambda\left(\nu \cdot\left[\varepsilon\left(e_{2}\right) a e_{3}\right]\right) \cdot S^{-1}\left(e_{1}\right) \\
& =\sum_{(e)} \lambda\left(\nu \cdot a e_{2}\right) \cdot S^{-1}\left(e_{1}\right)=\tilde{\lambda}(\nu \cdot a) .
\end{aligned}
$$

Next, let $h \in H$, then $h=S^{-1}(g)$, some $g \in H$. Thus

$$
\begin{aligned}
\tilde{\lambda}(\nu) \cdot h & =\tilde{\lambda}(\nu) \cdot S^{-1}(g)=\sum_{(e)} \lambda\left(\nu \cdot e_{2}\right) \cdot S^{-1}\left(e_{1}\right) S^{-1}(g) \\
& =\sum_{(e),(g)} \lambda\left(\nu \cdot \varepsilon\left(g_{2}\right) e_{2}\right) S^{-1}\left(e_{1}\right) S^{-1}\left(g_{1}\right) \\
& =\sum_{(e),(g)} \lambda\left(\nu \cdot S^{-1}\left(g_{3}\right) g_{2} e_{2}\right) S^{-1}\left(g_{1} e_{1}\right)
\end{aligned}
$$

using similar maps as in [6] this equals

$$
\sum_{(e)} \lambda\left(\nu \cdot S^{-1}(g) e_{2}\right) S^{-1}\left(e_{1}\right)=\tilde{\lambda}(\nu \cdot h) .
$$


Thus $\tilde{\lambda}$ is an $A \# H$-module map. And if $w \in W, h \in H$, then $\lambda(w \cdot h)=$ $w \cdot h$, hence

$$
\tilde{\lambda}(w)=\sum_{(e)}\left(w \cdot e_{2}\right) \cdot S^{-1}\left(e_{1}\right)=w \varepsilon(e)=w .
$$

That is, $\tilde{\lambda}$ is an $A \# H$-projection from $V$ to $W$.

(2) As in [13], we observe that for any ring $S$, if $V$ is an $(S, A \# H)$ bimodule, and $W$ is an $(S, A \# H)$-subbimodule of $V$, which is an $(S, A)$ direct summand then the above $\tilde{\lambda}$ can be used to show it is an $(S, A \# H)$ direct summand. Similar statements can be made about $(A \# H, S)$ bimodules. Thus the result follows as in [13].

2. Inner actions and separable subalgebras. In what follows we shall be mainly interested in inner actions of Hopf algebras. Let us first recall this notion which genralizes the known ones for automorphisms or derivations $[4,18]$.

Let $H$ be a Hopf algebra and $A$ an $H$-module algebra (less can be assumed on $A$ but we have no need for it here). We say that the action of $H$ on $A$ is inner if there exist $u, v \in \operatorname{Hom}_{k}(H, A)$ so that for all $h \in H$, $a \in A$ we have:

$$
\begin{gathered}
\sum_{(h)} u\left(h_{1}\right) \nu\left(h_{2}\right)=\sum_{(h)} u\left(h_{1}\right) u\left(h_{2}\right)=\varepsilon(h) \\
h \cdot a=\sum_{(h)} u\left(h_{1}\right) a \nu\left(h_{2}\right) .
\end{gathered}
$$

Sometimes (1), (2) hold with $u, \nu \in \operatorname{Hom}_{k}(H, B)$ where $A \subset B$ is a $k$-algebra. We say then that the action of $H$ on $A$ is $B$-inner. This generalizes the notion of $X$-inner automorphisms, or $Q$-inner derivations in which the "inducing elements" came from the quotient rings of $A$ rather than from $A$ itself.

In the following proposition we shall summarize some facts, most come from [4]. Relation (4) is new and essential for the proof of Proposition 12 .

Proposition 8. If the action of $H$ on $A$ is inner then:

(1) All ideals of $A$ are $H$-stable, hence the quotient rings $Q^{r}, Q^{l}$ are the usual Martindale quotients rings.

(2) $Z(A) \subset A^{H}$.

(3) $[4,18]$ For any $a \in A, h \in H$ we have

$$
u(h) a=\sum_{(h)}\left(h_{1} \cdot a\right) u\left(h_{2}\right), \quad a \nu(h)=\sum_{(h)} \nu\left(h_{1}\right)\left(h_{2} \cdot a\right)
$$


(4) For any $a \in A, h \in H$ we have

$$
\begin{aligned}
& \left.a u(h)=\sum_{(h)} u\left(h_{2}\right)\left(S^{-1} h_{1}\right) \cdot a\right) \quad\left(\text { provided } S^{-1} \text { exists }\right), \text { and } \\
& \nu(h) a=\sum_{(h)}\left(S\left(h_{2}\right) \cdot a\right) \nu\left(h_{1}\right)
\end{aligned}
$$

(5) [4] If $g, h \in H$ then

$$
t(g, h)=\sum_{(h),(g)} \nu\left(h_{1}\right) \nu\left(g_{1}\right) u\left(g_{2} h_{2}\right) \text { belongs to } Z(A) .
$$

(6) For $g, h \in H$ define $\mu(g, h)=\sum_{(g),(h)} \nu\left(g_{1} h_{1}\right) u\left(g_{2}\right) u\left(h_{2}\right)$, then

$$
(t * \mu)(h, g)=(\mu * t)(h, g)=\varepsilon(h g),
$$

that is, $\mu=t^{-1}$ under convolution *. In particular, $\mu(g, h) \in Z(A)$ for all $g, h \in H$.

Proof. We prove (4) the rest are easily checked. Let $a \in A, h \in H$ then

$$
\begin{aligned}
\sum_{(h)} u\left(h_{2}\right)\left(S^{-1}\left(h_{1}\right) \cdot a\right) & =\sum_{(h)}\left[h_{2} \cdot\left(S^{-1}\left(h_{1}\right) \cdot a\right)\right] u\left(h_{3}\right) \quad \text { (by part (3)) } \\
& =\sum_{(h)}\left(h_{2} S^{-1}\left(h_{1}\right) \cdot a\right) u\left(h_{3}\right)=\sum_{(h)} \varepsilon\left(h_{1}\right) a u\left(h_{2}\right) \\
& =a u(h) .
\end{aligned}
$$

The second claim follows similarly.

The function $t: H \times H \rightarrow Z(A)$ of Proposition 8 plays an important role in [4], where we showed that under the hypothesis of the proposition,

$$
A \# H \stackrel{\theta}{\cong} A_{t}[H] .
$$

Where $A_{t}[H]$ is the twisted product of $A$ with $H$ with 2-cocycle $t$. That is, $A_{t}[H]=A \otimes_{k} H$ as a vector space, but multiplication is defined by:

$(a \otimes h)(b \otimes g)=\sum_{(h)(g)} a b t\left(h_{1}, g_{1}\right) \otimes h_{2} g_{2}$

$$
\text { all } a, b \in A \text { and } h, g \in H \text {. }
$$

Note that $A_{t}[H]$ is a free left $A$-module with basis $\left\{1 \otimes h_{i}\right\}$ where $\left\{h_{i}\right\}$ is any $k$-basis for $H$. Also, $A$ is embedded naturally in $A_{t}[H]$ as $A \otimes 1$, we shall identify $A$ with its image. Under this identification, the elements $1 \otimes h$, where $h \in H$, commute with $A$. Finally, if Maschke theorem holds 
for $A \# H$ then it holds for $A_{t}[H]$ since $A$ is naturally embedded in both. To each $h \in H$ we associate $1 \otimes h \in A_{t}[H]$. However, one can also associate to it

$$
\theta(1 \# h)=\sum_{(h)} u\left(h_{1}\right) \otimes h_{2} \in A_{t}[H]
$$

( $\theta$ is the isomorphism between $A \# H$ and $A_{t}[H]$ ).

Let us denote $\theta(1 \# h)=\bar{h} \in A_{t}[H]$. Whereas

$$
\begin{aligned}
& (1 \otimes h)(1 \otimes g)=\sum_{(h)(g)} t\left(h_{1}, g_{1}\right) \otimes h_{2} g_{2}, \\
& \bar{h} \bar{g}=\theta(1 \# h) \theta(1 \# g)=\theta(1 \# h g)=\overline{h g} .
\end{aligned}
$$

Let us summarize some properties of ${ }^{-}$.

LEMMA 9. Let $\bar{h}$ be as above.

(1) If $\left\{h_{i}\right\}$ is a k-basis for $H$ then $\left\{\bar{h}_{i}\right\}$ is an A-basis for $A_{t}[H]$.

(2) $\bar{h} x=x \bar{h}$ for all $x \in A^{H}$.

(3) If $H$ is finite-dimensional and semisimple with $e$ the idempotent in the integral then $\bar{h} \bar{e}=\bar{e} \bar{h}=\varepsilon(h) \bar{e}$, and $\bar{e} A_{t}[H] \bar{e}=A^{H} \bar{e} \cong A^{H}$.

Proof. (1) If $\left\{h_{i}\right\}$ is a $k$-basis of $H$, then $\left\{1 \# h_{i}\right\}$ is an $A$-basis for $A \# H$. Since $\theta$ is an isomorphism $\left\{\theta\left(1 \# h_{i}\right)\right\}=\left\{\bar{h}_{i}\right\}$ is a $\theta(A)$-basis for $A_{t}[H]$. But $\theta(A)=A$, hence the result.

(2) The elements $1 \# h$ commute with $A^{H}$ in $A \# H$, hence $\bar{h}=\theta(1 \# h)$ commutes with $A^{H}$.

(3) follows similarly from $A \# H$, for which the above hold by [6].

Studying groups acting as automorphisms on division rings E. Noether [8] introduced the so called algebra of the group. This notion was since generalized to semiprime rings [13]. The algebra of the group "mirrors" $k G$ in $A$ when $G$ acts as inner automorphisms on $A$. Analogously, we shall define an algebra $B$ for inner actions of Hopf algebras. Let $A$ be an $H$-module algebra, and assume the action of $H$ on $A$ is inner, induced by $u, \nu$ with $u(1)=\nu(1)=1$ (this can always be assumed).

Let $V=\{\nu(h) \mid h \in H\} \subset A$ and let $Z=$ center of $A$. Let $B$ denote the $Z$-module generated by $V$. Observe that since $\nu(1)=1,1 \in B$. We show next that $B$ is actually a $Z$-algebra.

Lemma 10. Let $A$ be an $H$-module algebra, and assume the action of $H$ on $A$ is inner. Let $B, u, \nu, Z$ be as above, then

(1) $B$ is Z-algebra.

(2) For any $\phi \neq T \subset A$ we have:

$$
C_{T}(B)=T^{H} \text {. }
$$


Proof. (1) Let $x, y \in V$, we show that $x y \in B$. Well $x=\nu(g)$, $y=\nu(h)$, for some $g, h \in H$, and hence

$$
\begin{aligned}
x y & =\nu(g) \nu(h)=\sum_{(g)(h)} \nu\left(g_{1}\right) \nu\left(h_{1}\right) \varepsilon\left(h_{2}\right) \varepsilon\left(g_{2}\right) \\
& =\sum_{(g)(h)} \nu\left(g_{1}\right) \nu\left(h_{1}\right) \varepsilon\left(h_{2} g_{2}\right) \\
& =\sum_{(g)(h)} \nu\left(g_{1}\right) \nu\left(h_{1}\right) u\left(h_{2} g_{2}\right) \nu\left(h_{3} g_{3}\right) \\
& =\sum_{(g)(h)} t\left(h_{1}, g_{1}\right) \nu\left(h_{2} g_{2}\right) .
\end{aligned}
$$

By Proposition $8, t$ has values in $Z$, hence $x y \in Z V=B$.

(2) Let $x \in C_{T}(B)$, then for any $h \in H$,

$$
h \cdot x=\sum_{(h)} u\left(h_{1}\right) x \nu\left(h_{2}\right)=\sum_{(h)} u\left(h_{1}\right) \nu\left(h_{2}\right) x=\varepsilon(h) x .
$$

Hence $x \in T^{H}$.

Conversely, let $x \in T^{H}$. Then all we must show is that $x \nu(h)=\nu(h) x$ for each $h \in H$. Well, by Proposition 8, $x \nu(h)=\sum_{(h)} \nu\left(h_{1}\right)\left(h_{2} \cdot x\right)$. But since $x \in A^{H}$, this equals $=\sum_{(h)} \nu\left(h_{1}\right) \varepsilon\left(h_{2}\right) x=\nu(h) x$.

We consider next homomorphic images. If $I$ is an $H$-stable ideal of $A$ then $H$ acts on $\bar{A}=A / I$ in the obvious manner, that is, $h \cdot \bar{a}=\overline{h \cdot a}$, all $h \in H, a \in A$. If the action of $H$ on $A$ is inner then every ideal $I$ is $H$-stable.

Lemma 11. Let $A$ be an $H$-module algebra, and $I \neq A$, an $H$-stable ideal of $A$. Then,

(1) if the action of $H$ on $A$ is inner, then the action of $H$ on $\bar{A}$ is inner.

(2) if $H$ is finite-dimensional and semisimple (no inner action assumed), then

$$
\overline{A^{H}}=\overline{A^{H}} .
$$

Proof. (1) Let $u, \nu \in \operatorname{Hom}_{k}(H, A)$ induce the action of $H$ on $A$. Let $\bar{u}, \bar{\nu} \in \operatorname{Hom}_{k}(H, \bar{A})$ by: $\bar{u}(h)=\overline{u(h)}, \bar{\nu}(h)=\overline{\nu(h)}$, all $h \in H$. Then for all $h \in H, \bar{a} \in \bar{A}$ :

$$
h \cdot \bar{a}=\overline{h \cdot a}=\sum_{(h)} \overline{u\left(h_{1}\right) a \nu\left(h_{2}\right)}=\sum_{(h)} \overline{u\left(h_{1}\right)} \bar{a} \overline{\nu\left(h_{2}\right)}=\sum \bar{u}\left(h_{1}\right) \bar{a} \bar{\nu}\left(h_{2}\right) .
$$

Since $I \neq A, \bar{A}$ contains the identity $\overline{1}$ and hence for each $h \in H$,

$$
\sum_{(h)} \bar{u}\left(h_{1}\right) \bar{\nu}\left(h_{2}\right)=\sum_{(h)} \overline{u\left(h_{1}\right) \nu\left(h_{2}\right)}=\varepsilon(h) \overline{1} .
$$


We have shown that $\bar{u}, \bar{\nu}$ induce the action of $H$ on $A$, thus this action is inner.

(2) Since $H$ is semisimpole and finite-dimensional, $H$ contains the idempotent integral $e$, and, $A^{H}=e \cdot A$. Thus

$$
\overline{A^{H}}=\overline{e \cdot A}=e \cdot \bar{A}=\bar{A}^{H} .
$$

As for automorphisms and derivations, we shall deal with $Q$-inner actions. We prove that just as for $X$-inner automorphisms, the "inducing elements" originally assumed in $Q^{r}$ or $Q^{l}$ necessarily come from the smaller $Q^{s}$.

Proposition 12. Let $H$ have bijective antipode. Let $A$ be an $H$-module algebra and assume the action of $H$ on $A$ is $Q^{r}$ or $Q^{l}$-inner, then it is $Q^{s}$-inner. That is, for each $h \in H$ there exists an $I \in \mathscr{F}$ so that all of $u(h) I, \nu(h) I, I u(h)$ and $I \nu(h)$ are contained in $A$.

Proof. Say the action of $H$ on $A$ is $Q^{r}$-inner. That is $u, \nu \in$ $\operatorname{Hom}_{k}\left(H, Q^{r}\right)$. Let $h \in H$, with $\Delta(h)=\sum_{(h)} h_{1} \otimes h_{2}$. Since this expression involves only a finite number of elements in $H$, there exists an $I \in \mathscr{F}$ so that:

$$
u\left(h_{2}\right) I \subset A \text { and } \nu\left(h_{1}\right) I \subset A, \text { for all } h_{1}, h_{2}
$$

mentioned above.

Now let $a \in I$, then by Proposition 8.4

$$
a u(h)=\sum_{(h)} u\left(h_{2}\right)\left(S^{-1}\left(h_{1}\right) \cdot a\right) .
$$

But since $I$ is $H$-stable and since $u\left(h_{2}\right) I \subset A$, all $h_{2}$, it follows that $a u(h) \subset A$. We have shown that $I u(h) \subset A$. Now, $u(h) I=$ $\sum_{(h)} \varepsilon\left(h_{1}\right) u\left(h_{2}\right) I$, hence $u(h) I \subset A$. The statement about $\nu(h)$ follows similarly from Proposition 8.3, and the one for $Q^{l}$-inner actions is proved analogously.

In view of Proposition 12, we say that the action of $H$ on $A$ is $Q$-inner, if it is $Q^{r}, Q^{l}$ or $Q^{s}$ inner.

In the following lemma we show that inner actions on $A$ induce inner actions on $Q$.

LEMMA 13. Let $H$ have bijective antipode. If the action of $H$ on $A$ is $Q$-inner then the action of $H$ on $Q^{r}$ and $Q^{l}$ is inner.

Proof. We prove it for $Q^{r}$, the proof for $Q^{l}$ is similar. Let $u, \nu \in$ $\operatorname{Hom}_{k}(H, Q)$ induce the action of $H$ on $A$. Let $q \in Q^{r}$, and let $I \in \mathscr{F}$ so 
that $q: I \rightarrow A$. Let $a \in I$, then we claim that:

$$
(h \cdot q)(a)=\left[\sum_{(h)} u\left(h_{1}\right) q v\left(h_{2}\right)\right] a .
$$

Hence $h \cdot q=\sum_{(h)} u\left(h_{1}\right) q \nu\left(h_{2}\right)$. That is $u, \nu$ induce the action of $H$ on $Q^{r}$. Well,

$$
\begin{aligned}
(h \cdot q)(a) & =\sum_{(h)} h_{1} \cdot\left[q\left(S\left(h_{2}\right) \cdot a\right)\right] \\
= & \sum_{(h)} u\left(h_{1}\right) q\left[S\left(h_{3}\right) \cdot a\right] \nu\left(h_{2}\right) \\
= & \sum_{(h)} u\left(h_{1}\right) q\left[\left(S\left(h_{3}\right) \cdot a\right) \nu\left(h_{2}\right)\right] \quad(q \text { is a right } A \text {-module map) } \\
= & \sum_{(h)} u\left(h_{1}\right) q\left[\nu\left(h_{2}\right)\left(h_{3} S\left(h_{4}\right) \cdot a\right)\right] \quad \text { (Proposition 8.3) } \\
= & \sum_{(h)} u\left(h_{1}\right) q\left[\nu\left(h_{2}\right) \varepsilon\left(h_{3}\right) a\right] \\
= & {\left[\sum_{(h)} u\left(h_{1}\right) q \nu\left(h_{2}\right)\right] a . }
\end{aligned}
$$

Let us specialize now to $Q$-inner actions when $A$ is $H$-prime. Let us recall that a prime ring $R$ is called centrally closed if it contains $C$, the center of the Martindale ring of quotients of $R$, called the extended centroid of $R$. This center is a field, and it consists of $(R, R)$-bimodule homomorphisms.

Just as for the Martindale ring of quotient it is easily seen that every non-zero $(A, A)$-bimodule of $Q^{r}\left(Q^{l}\right.$, or $\left.Q^{s}\right)$ intersect $A$ nontrivially. Hence if $A$ is $H$-prime or $H$-semiprime then so is $Q^{r}\left(Q^{l}\right.$ or $\left.Q^{s}\right)$.

The following generalizes a result of [5], the proof is essentially the same, using Lemma 10 and the above observation. The same statements can be made about $Q^{l}$ or $Q^{r}$, we denote $Q^{s}=Q$.

Proposition 14. Let $A$ be $H$-prime. If the action of $H$ on $A$ is $Q$-inner, then $Q$ is prime and centrally closed. In particular, the center of $Q$ is a field.

Proof. By Lemma 13, the action of $H$ on $Q$ is inner. Thus, every ideal of $Q$ is $H$-stable. Since $Q$ is also $H$-prime, it is prime, and $Q^{r}(Q)=$ Martindale ring of quotients of $Q$. By Lemma 13, the action of $H$ is inner on $Q^{r}(Q)$, hence in particular, the action of $H$ on the extended centroid, $C$, is trivial. Let $0 \neq z \in C$, then there exists an ideal of $Q$ such that 
$0 \neq z I=I z \subset Q$. Since $z I$ is an ideal of $Q$, we have $0 \neq z I \cap A$. Let

$$
J=\{x \in I \mid x z \in A\}
$$

then $0 \neq J \subset I$ is obviously an $(A, A)$-bimodule of $Q$; moreover it is $H$-stable, since $H$ acts trivially on $C$. Let

$$
T=J \cap A \neq 0
$$

then $T$ is an $H$-stable ideal of $A$ contained in $I$. The restriction of $z$ to $T$ yields an element of the center of $Q$, hence $z \in Z(Q)$. We have shown that $C \subset Z(Q)$, and thus $C=Z(Q)$. Combining this with Lemma 10 applied to $Q$, we have:

Corollary 15. If $A$ is $H$-prime and the action of $H$ on $A$ is $Q$-inner, then $B$ is an algebra over the field $C$, and $\operatorname{dim}_{C} B \leq \operatorname{dim}_{k} H$.

Much more can be said if $H$ is assumed to be finite-dimensional and semisimple. For then, as for automorphisms [13], we show that $B$ is a finite-dimensional separable $C$-subalgebra of $Q=Q^{s}$.

Proposition 16. Let $H$ be an $n$-dimensional, semisimple Hopf algebra, $A$ an $H$-prime, $H$-module algebra. If the action of $H$ on $A$ is $Q^{r}$-inner then $B$ is an m-dimensional separable $C$-subalgebra of $Q$, with $m \leq n$. If moreover $A$ is prime and the action is inner then $C$ is the usual extended centroid of $A$, and $B \subset A C$.

Proof. By Proposition $12 Q^{r}$-inner actions are $Q$-inner actions. By Lemma 13, the action of $H$ on $Q$ is inner. Let $\left\{h_{i}\right\}, i=1, \ldots, n$, be a basis for $H$, then $\left\{1 \otimes h_{i}\right\}$ is a $Q$-basis of $Q_{t}[H]$. Recall that

$$
(1 \otimes h)(1 \otimes g)=\sum_{(h)(g)} t\left(h_{1}, g_{1}\right) \otimes h_{2} g_{2},
$$

where $t(h, g) \in Z(Q)=C$, a field (by Proposition 14). Let $E$ be the vector space over $C$,

$$
E=\operatorname{Span}_{C}\{1 \otimes h \mid h \in H\}
$$

then by above, $E$ is a $C$ subalgebra of $Q_{t}[H]$ of dimension $n$. As a matter of fact,

$$
E=C_{t}[H] \text { and } Q_{t}[H]=Q E=E Q .
$$

The last equality holds since $\{(1 \otimes h)\}$ and $C$ both centralize $Q$. We first show that $E$ is a separable $C$-algebra. Let $k \subset C \subset L$, we show that

$$
E \otimes_{C} L=C_{t}[H] \otimes_{C} L
$$


is semisimple. Well, since the action of $H$ on $Q$ is inner, and $Q$ is prime, [4] implies that $Q_{t}[H]$ is semiprime. Since $Q_{t}[H]=Q E$ and since $E$ centralizes $Q, E$ must be semiprime. However $E$ is finite-dimensional, hence semisimple. The following shows that extending $C$ by $L$ reduces to the above situation.

Let $K=H \otimes_{k} L$, then $K$ is an $n$-dimensional semisimple Hopf algebra over $L$ [3]. Let $T=Q \otimes_{C} L$, we show that $T$ is a prime $K$-module algebra, that the action of $K$ on $T$ is inner, that $L_{t}[K] \subset T_{t}[K]$ serves the role of $C_{t}[H]$ in $Q_{t}[H]$ hence as above $L_{t}[K]$ is semisimple. We finally show that $L_{t}[K] \cong E \otimes_{C} L$ and thus deduce that $E$ is separable.

First, $K$ acts on $T$ by:

$$
\left(h \otimes_{k} l\right) \cdot\left(q \otimes_{C} m\right)=h \cdot q \otimes_{C} l m, \text { for all } h \in H, q \in Q, l, m \in L \text {. }
$$

This action is well defined since the action of $H$ on $Q$ is inner, hence $H$ acts trivially on $C$, and thus $h \cdot(q c)=(h \cdot q) c$. As is easily checked, this action is inner induced by the "extended" $u^{\prime}, \nu^{\prime} \in \operatorname{Hom}_{L}(K, T)$, defined by:

$$
u^{\prime}(h \otimes l)=u(h) \otimes l, \nu^{\prime}(h \otimes l)=\nu(h) \otimes l \quad \text { for all } h \in H, l \in L .
$$

For completeness let us check that

$$
\sum_{(k)} u^{\prime}\left(k_{1}\right) \nu^{\prime}\left(k_{2}\right)=\varepsilon(k), \quad \text { all } k \in K
$$

Well, recall [3] that for $k=h \otimes_{k} l \Delta(k)=\sum_{(h)}\left(h_{1} \otimes_{k} l\right) \otimes_{L}\left(h_{2} \otimes 1\right)$ and $\varepsilon(k)=\varepsilon(h) l$. Hence

$$
\begin{aligned}
\sum_{(k)} u^{\prime}\left(k_{1}\right) \nu^{\prime}\left(k_{2}\right) & =\sum_{(h)} u^{\prime}\left(h_{1} \otimes l\right) \nu^{\prime}\left(h_{2} \otimes 1\right) \\
& =\sum_{(h)}\left(u\left(h_{1}\right) \otimes l\right)\left(\nu\left(h_{2}\right) \otimes 1\right) \\
& =\left[\sum_{(h)} u\left(h_{1}\right) \nu\left(h_{2}\right)\right] \otimes l=\varepsilon(h) l=\varepsilon(k) .
\end{aligned}
$$

Let $t^{\prime}$ relate to $u^{\prime}, \nu^{\prime}$ as does $t$ to $u, \nu$. It is easy to check that; for all $h, g \in H, l, m \in L$ we have:

$$
t^{\prime}(h \otimes l, g \otimes m)=t(h, g) \otimes l m .
$$

that is, $t^{\prime}$ extends $t$, thus we shall identify $t^{\prime}$ with $t$. Now, $T=Q \otimes_{C} L$ is prime, and the extended centroid of $T$ is $L$. This follows from the fact that $Q$ is prime and centrally closed (by Proposition 14), and from an argument in [11, Theorem 2.2]. Thus,

$$
T_{t}[K]=T L_{t}[K]
$$


Since $T$ is prime, $T_{t}[K]$ is semiprime [4], hence $L_{t}[K]$ is semiprime. Finally to show that $L_{t}[K] \cong C_{t}[H] \otimes_{C} L$, the obvious correspondence:

$$
\phi:\left(H \otimes_{k} L\right) \otimes_{L} L \rightarrow\left(H \otimes_{k} C\right) \otimes_{C} L
$$

by

$$
h \otimes l \otimes m \rightarrow h \otimes 1 \otimes l m
$$

for all $h \in H, l, m \in L$ is easily seen to be an algebra isomorphism. We have shown that $E$ is an $n$-dimensional separable algebra over $C$. Now define for all $c \in C, h \in H$,

$$
\psi: E \rightarrow B \text { by } c \otimes h \rightarrow c \nu(h) .
$$

Extending linearly to $E$.

This is clearly a $C$-linear surjection. We show it is an algebra antihomomorphism. Let $h, g \in H$, then

$$
\begin{aligned}
\psi[(1 \otimes h)(1 \otimes g)] & =\psi\left[\sum_{(h)(g)} t\left(h_{1}, g_{1}\right) \otimes h_{2} g_{2}\right] \\
& =\sum_{(h),(g)} t\left(h_{1}, g_{1}\right) \nu\left(h_{2} g_{2}\right)=\nu(g) \nu(h) \quad \text { (Lemma 10) } \\
& =\psi(1 \otimes g) \psi(1 \otimes h) .
\end{aligned}
$$

We have shown that $B$ is an anti-homomorphic image of an $n$-dimensional separable $C$-algebra, hence $B$ is a separable $C$-algebra of dimension less than or equal to $n$.

REMARK. The above proof is in the same vein as the ones used for automorphisms [12, 13]. A basic ingredient in these arguments is a Maschke theorem for twisted group rings $F_{\tau}[G]$, which states that if $F$ is a field with $|G|^{-1} \in F$ then $F_{\tau}[G]$ is semisimple. An analogous result for Hopf algebras is yet unknown. In the course of the proof of Proposition 16 we showed that for an inner action on $Q$, the result holds with $F=L \supset C$, and $\tau=t$.

Combining Proposition 16, Lemma 11 and [12, Theorems 1, 2]. We prove a generalization of [12, Theorem 3]. The method of proof is analogous to that of [12].

THEOREM 17. Let $H$ be an $n$-dimensional semisimple Hopf algebra over $k$. Let $A$ be an $H$-module algebra, and assume the action of $H$ on $A$ is inner. If $A^{H}$ satisfies a polynomial identify of degree $m$ then $A$ satisfies $\left(s_{n m}[x]\right)^{t}$, some integer $t$. If $A$ is semiprime then $t=1$. 
Proof. Note that since the action of $H$ is inner, every ideal of $A$ is $H$-stable. First assume $A$ is prime. Then by Proposition $16, B \subset A C$ is a finite-dimensional separable subalgebra over $C$, and $\operatorname{dim}_{C} B \leq n$. By Lemma 10

$$
C_{A C}(B)=(A C)^{H}=A^{H} C \text {. }
$$

The last equality holds since $H$ acts trivially on $C$. Since $A^{H}$ is P.I. so is $A^{H} C$, hence so is $C_{A C}(B)$. Thus we are in the situation of [12] where we have a finite-dimensional separable subalgebra whose centralizer satisfies a P.I. and we conclude by [12] that $A C$ satisfies $s_{m n}[x]$. In particular, $A$ satisfies $s_{m n}[x]$.

If $A$ is semiprime, then $A$ is a subdirect sum of prime images. By Lemma 11, all hypotheses are preserved by these images, hence each satisfies, by the above, $s_{m n}[x]$. The same is then true for $A$. The rest now follows by an argument in [1] as adapted by [12].

Another application of Proposition 16 is to question about integrality. Let us recall the definition [14]. Let $R \supset S$ be a ring extension. An $S$-monomial in $r_{1}, \ldots, r_{m} \in R$ is a product in some order of the $r_{i}$ 's, each occurring finitely often, and of elements of $S$ with at least one element of $S$ occurring. The extension $R \supset S$ is said to be fully integral of degree $m$ if for any $r_{1}, \ldots, r_{m} \in R$

$r_{1} r_{2} \cdots r_{m}=\phi\left(r_{1}, \ldots, r_{m}\right)$ where $\phi\left(r_{1}, \ldots, r_{m}\right)$ is a sum of $S$-monomials of degree less than $m$.

THEOREM 18. Let $H$ be an $n$-dimensional semisimple Hopf algebra. Let $A$ be a prime $H$-module algebra, and assume that action of $H$ on $A$ is inner. Then $A$ is fully integral over $A^{H} C$ of degree $t \leq 2^{2^{n+1}}$ (where $C$ is the extended centroid of $A$ ).

Proof. By Proposition 16, $A C$ contains $B$, an $m$-dimensional separable $C$-subalgebra of $A C$ with $m \leq n$. By Lemma $10, C_{A C}(B)=(A C)^{H}=$ $A^{H} C$. Thus, by [14, Theorem 6], $A C$ is fully integral over $A^{H} C$ of degree $t \leq 2^{2^{m+1}} \leq 2^{2^{n+1}}$. This is true in particular for $A$ over $A^{H} C$.

There are some immediate corollaries to the above theorem.

Corollary 19. Let $H$ be as in Theorem 18, and assume that $A$ is a finite direct sum of simple algebras. If the action of $H$ on $A$ is inner, then $A$ is fully integral over $A^{H}$ of degree $t \leq 2^{2^{n+1}}$.

Proof. By hypothesis

$$
A=A_{1} \oplus A_{2} \oplus \cdots \oplus A_{m}
$$


where $A_{i}=e_{i} A, e_{i}$ is a primitive central idempotent of $A$ and $\left\{e_{i}\right\}$ are orthogonal. Since each $A_{i}$ is an ideal of $A$, and since the action is inner, each $A_{i}$ is $H$-stable and $H$ acts trivially on $e_{i}$. Moreover, the action of $H$ on $A_{i}$ is inner induced by

$$
u^{\prime}, \nu^{\prime} \in \operatorname{Hom}_{k}\left(H, A_{i}\right), \quad u^{\prime}(h)=u(h) e_{i}, \quad \nu^{\prime}(h)=\nu(h) e_{i} .
$$

To see this, let $x \in A_{i}$, then $x=a e_{i}$. Let $h \in H$, then

$$
\begin{aligned}
h \cdot x & =h \cdot\left(a e_{i}\right)=(h \cdot a) e_{i}=\sum_{(h)} u\left(h_{1}\right) a \nu\left(h_{2}\right) e_{i} \\
& =\sum_{(h)} u\left(h_{1}\right) e_{i} a e_{i} \nu\left(h_{2}\right) e_{i}=\sum_{(h)} u^{\prime}(h) x \nu^{\prime}(h) .
\end{aligned}
$$

Thus each $A_{i}$ is a simple algebra with 1 on which $H$ acts innerly. Since in this case $C \subset A_{i}$ we have by Theorem 17 that $A_{i}$ is fully integral over $A_{i}^{H}$ of degree $t \leq 2^{2^{n+1}}$. However, $A_{i}^{H}=\left(e_{i} A\right)^{H}=e_{i} A^{H} \subset A^{H}$. Hence each $A_{i}$ is fully integral of degree $t$ over $A^{H}$, and consequently the same is true about their sum, $A$.

In the next section (Proposition 22) we prove some more properties of $A$ satisfying the conditions in the corollary.

3. Further consequences of Maschke's theorem. We assume as before that $H$ is $n$-dimensional and semisimple and that the action of $H$ on $A$ is inner. Recall, that $A \# H \cong A_{t}[H]$, and that by Proposition 7 the left, right or two-sided Maschke theorem holds for $A \# H$, and hence for $A_{t}[H]$.

We proceed as in [10] to relate modules over $A, A^{H}$ and $A_{t}[H]$.

Let $V$ be a right $A$-module, then the induced $A_{t}[H]$-module $\bar{V}$ given by:

$$
\bar{V}=V \otimes_{A} A_{t}[H]=\sum_{i=1}^{n} V \otimes_{A} h_{i},
$$

where $\left\{h_{i}\right\}_{i=1}^{n}$ is a $k$-basis for $H$, chosen so that $h_{1}=1$. Each $V \otimes h_{i}$ is an $A$-module, for $h_{i}$ commutes with $A$ in $A_{t}[H]$, and is isomorphic as an $A$-module to $V \otimes 1$. It is this property of $\bar{V}$ which enabled us in [4] to prove that $A \# H$ is semiprime, and which is also a key to what follows. A notation first. For any $R$-module $M_{R}$, denote by $\mathscr{L}\left(M_{R}\right)$ the lattice of right $R$-submodules of $M$. Back to $V_{A}$ as above, $V$ is an $A^{H}$-module under restriction. We use the notation and consequences of Lemma 9. In particular, each element $w \in \bar{V}$ can be written uniquely 
as $w=\sum_{i=1}^{n} \nu_{i} \otimes \bar{h}_{i}, \nu_{i} \in V, h_{\imath} \in H$. Define:

$\tau: \mathscr{L}\left(\bar{V}_{A_{t}[H]}\right) \rightarrow \mathscr{L}\left(V_{A^{H}}\right), \quad$ by $w \rightarrow \sum_{i=1}^{n} \nu_{l} \varepsilon\left(h_{i}\right)$,

any $w \in X, X \in \mathscr{L}(\bar{V})$

$\sigma: \mathscr{L}\left(V_{A^{H}}\right) \rightarrow \mathscr{L}\left(\bar{V}_{A_{t}[H]}\right), \quad$ by $U \rightarrow\left(U \otimes_{A} \bar{e}\right) A_{t}[H]$,

$$
\text { any } U \in \mathscr{L}\left(V_{A^{H}}\right) \text {. }
$$

Since $\bar{h}_{i}$ commutes with $A^{H}, \tau$ is a right $A^{H}$-module map. Thus, if $X \in \mathscr{L}\left(\bar{V}_{A_{t}[H]}\right), X^{\tau} \in \mathscr{L}\left(V_{A^{H}}\right)$. Moreover, note that for any $w \in \bar{V}$

$$
\begin{aligned}
w \bar{e} & =\sum_{i=1}^{n} \nu_{i} \otimes \overline{h_{i} e}=\sum_{i} \nu_{l} \otimes \varepsilon\left(h_{i}\right) \bar{e} \\
& =\left(\sum_{i} \nu_{i} \varepsilon\left(h_{l}\right)\right) \otimes \bar{e}=w^{\tau} \otimes \bar{e} .
\end{aligned}
$$

LEMma 20. Let $H$ be an $n$-dimensional semisimple Hopf algebra acting innerly on $A$, an $H$-module algebra. Let $V_{A}, \sigma, \tau$ be as above then:

(1) For $U \in \mathscr{L}\left(V_{A^{H}}\right), U^{\sigma \tau}=U$. Thus, $\sigma$ is strictly increasing.

(2) For $X_{1}, X_{2} \in \mathscr{L}\left(\bar{V}_{A_{t}[H]}\right)$,

$$
\left(X_{1} \oplus X_{2}\right)^{\tau}=X_{1}^{\tau} \oplus X_{2}^{\tau} \text {. }
$$

Proof. (1) Let $U \in \mathscr{L}\left(V_{A^{H}}\right)$, then

$$
\begin{aligned}
U^{\sigma} \bar{e} & =\left(U \otimes_{A} \bar{e}\right) A_{t}[H] \bar{e}=U \otimes_{A} \bar{e} A_{t}[H] \bar{e} \\
& =U \otimes_{A} A^{H} \bar{e} \quad(\text { by Lemma } 9) \\
& =U \otimes_{A} \bar{e}
\end{aligned}
$$

However, as noted above, for any $w \in \bar{V}, w \bar{e}=w^{\tau} \otimes \bar{e}$. Thus, $U^{\sigma} \bar{e}=$ $U^{\sigma \tau} \otimes_{A} \bar{e}$. We have shown that $U \otimes_{A} \bar{e}=U^{\sigma} \bar{e}=U^{\sigma \tau} \otimes_{A} \bar{e}$ and hence that $U=U^{\sigma \tau}$.

(2) It is clear that $\left(X_{1}+X_{2}\right)^{\tau}=X_{1}^{\tau}+X_{2}^{\tau}$.

If $X_{1} \cap X_{2}=0$, then in particular,

$$
X_{1} \bar{e} \cap X_{2} \bar{e}=0 \text {. }
$$

By the above remark, $X_{l} \bar{e}=X_{l}^{\tau} \otimes_{A} \bar{e}, i=1,2$. Hence

$$
0=\left(X_{1}^{\tau} \otimes_{A} \bar{e}\right) \cap\left(X_{2}^{\tau} \otimes_{A} \bar{e}\right)=\left(X_{1}^{\tau} \cap X_{2}^{\tau}\right) \otimes_{A} \bar{e},
$$

Hence $X_{1}^{\tau} \cap X_{2}^{\tau}=0$, and consequently $\left(X_{1} \otimes X_{2}\right)^{\tau}=X_{1}^{\tau} \oplus X_{2}^{\tau}$. 
We are ready to prove analogs of [10] relating the module structure of $V_{A}, V_{A^{H}}$ and $\bar{V}_{A_{t}[H]}$. By the radical of $V_{A}$ we mean the intersection of all maximal submodules of $V_{A}$, and $K \operatorname{dim} V_{A}$ denotes the Krull dimension.

THEOREM 21. Let $H$ be an n-dimensional semisimple Hopf algebra. Let $A$ be an $H$-module algebra and assume that the action of $H$ on $A$ is inner. Let $V$ be a right $A$-module, then:

(1) $V_{A}$ is Noetherian if and only if $V_{A^{H}}$ is Noetherian.

(2) if $V_{A}$ has finite length $m$, then $V_{A^{H}}$ has length $\leq m n$.

(3) if $V_{A}$ is completely reducible then $V_{A^{H}}$ is completely reducible.

(4) $\operatorname{rad} V_{A} \supset \operatorname{rad} V_{A^{H}}$

(5) $K \operatorname{dim} V_{A}$ exists if and only if $K \operatorname{dim} V_{A^{H}}$ exists, and then they are equal.

Proof. The proofs are essentially the same as the ones in [10], using Lemma 19 and the structure of $\bar{V}$. We shall prove (1) as an example. Recall that

$$
\bar{V}=\sum_{i=1}^{n} \otimes_{A} \otimes_{i}
$$

where $V \otimes_{A} h_{i}$ is isomorphic as an $A$-module to $V \otimes_{A} 1$. If $V_{A}$ is Noetherian then $V \otimes_{A} 1$ is a Noetherian $A$-module, and hence each $V \otimes_{A} h_{i}$ is a Noetherian $A$-module. Since $\bar{V}$ is a finite sum of Noetherian $A$-modules, $\bar{V}_{A}$ is a Noetherian $A$-module. Since $A \subset A_{t}[H], \bar{V}_{A_{t}[H]}$ is a Noetherian $A_{t}[H]$-module. Since

$$
\text { o: } \mathscr{L}\left(V_{A^{H}}\right) \rightarrow \mathscr{L}\left(\bar{V}_{A_{t}[H]}\right)
$$

is strictly increasing, the above implies that $V_{A^{H}}$ is Noetherian.

Along the same lines, we can use the two-sided version of Maschke theorem (Proposition 7) for $\left(A_{t}[H], A_{t}[H]\right)$-bimodules. This is analogous to [13, Theorem 7.9].

Proposition 22. Let $A, H$ be as in Theorem 21.

(1) if $V$ is an $\left(A_{t}[H], A_{t}[H]\right)$-bimodule which is a direct sum of $m$ simple $(A, A)$-bimodules, then $V$ is a direct sum of $\leq m$ simple ( $\left.A_{t}[H], A_{t}[H]\right)$-bimodules.

(2) if $A$ is a direct sum of $m$ simple algebras, then both $A_{t}[H]$ and $A^{H}$ are a direct sum of $\leq \mathrm{nm}$ simple algebras.

Proof. (1) Since $V$ is a completely reducible $(A, A)$-bimodule, Proposition 7 implies that $V$ is a completely reducible $\left(A_{t}[H], A_{t}[H]\right)$-bimodule; clearly the number of summands is $\leq m$. 
(2) If $A$ is a direct sum of $M$ simple algebras, then the same is true for each summand $A \otimes_{k} h_{i}$ in $A_{t}[H]$. Thus by part (1) $V=A_{t}[H]$ is a direct sum of $\leq m$ simple $\left(A_{t}[H], A_{t}[H]\right)$-bimodules. These are simple subalgebras, hence $A_{t}[H]$ is a direct sum of $\leq m n$ simple subalgebra. The same is true for $A^{H}$ since, by Lemma $9, A^{H} \cong \bar{e} A_{t}[H] \bar{e}$.

There are situations in which inner actions are guaranteed. Sweedler [18] has proved a generalization of the Noether-Skolem theorem to certain Hopf algebras. He has shown that if $H$ is a cocommutative Hopf algebra, all of whose simple subcoalgebras are 1-dimensional (i.e. pointed), and if $A$ is a central simple finite-dimensional algebra over $k$, which is an $H$-module algebra, then the action of $H$ on $A$ is inner. Applying the results of the previous sections we can describe some properties of $A$.

THEOREM 23. Let $H$ be an $n$-dimensional semisimple cocommutative Hopf algebra. Let $A$ be a central simple $n$-dimensional $H$-module algebra over $k=Z(A)$. Then

(1) $A$ is fully integral over $A^{H}$ of degree $t \leq 2^{2^{n+1}}$.

(2) $A$ is a finite $A^{H}$-module.

(3) $A^{H}$ is a direct sum of $\leq n$ simple algebras.

(1) Let $\bar{k}$ be the algebraic closure of $k$, then $\bar{H}=H \otimes_{k} \bar{k}$ is pointed [19], and $\bar{A}=A \otimes_{k} \bar{k}$ is central simple over $\bar{k}$, hence by [18], the action of $\bar{H}$ on $\bar{A}$ is inner. By Corollary $19, \bar{A}$ is fully integral over $\bar{A}^{\bar{H}}$, which by [3, Corollary 2] implies (1).

(2) Since $\bar{A}$ is finite-dimensional over $\bar{k}, V_{\bar{A}}=\bar{A}_{\bar{A}}$ has finite length. By Theorem 21 , this implies that $\bar{A}_{\bar{A}^{\bar{H}}}$ has finite length. However, $\overline{A^{H}}=\overline{A^{H}}$ [3], hence $A_{A^{H}}$ has finite length.

(3) is a consequence of Proposition 22.2 with $m=1$.

We conclude with an application.

COROLlaRy 24. Let $k$ have characteristic $p$, and let $L$ be $n$-dimensional semisimple restricted Lie algebra over $k$, acting as derivations on $A, a$ central simple finite-dimensional $k$-algebra. Then, $A^{L}$ is a direct sum of $\leq p^{n}$ simple algebras.

Proof. Let $H=u(L)$, the universal enveloping algebra of $L$. Then $\operatorname{dim}_{k} H=p^{n}, H$ is cocommutative and pointed and by assumption it is semisimple hence the result follows from Theorem 23.3.

Part (1) of Theorem 23 holds for $u(L)$ as above without any assumption on $A$, and part (2) holds when $A$ is only assumed to be semisimple artinian [3]. The considerations in [3] are different from the ones above. 


\section{REFERENCES}

[1] S. A. Amitsur, Rings with involution, Israel J. Math., 6 (1968), 99-106.

[2] J. Bergen and S. Montgomery, Smash products and outer derivations, Israel J. Math., (to appear).

[3] J. Bergen and M. Cohen, Actions of commutative Hopf algebras, to appear.

[4] R. J. Blattner, M. Cohen and S. Montgomery, Crossed products and inner actions of Hopf algebras, (to appear).

[5] W. Chin, Prime ideals in differential operator rings and crossed products of infinite groups, J. Algebra, (to appear).

[6] M. Cohen and D. Fishman, Hopf algebra and actions, J. Algebra, (to appear).

[7] M. Cohen, Hopf algebras acting on semiprime algebras, Contemporary Math., (to appear).

[8] N. Jacobson, Structure of rings, A.M.S. Colloquium Publ., 37 (1964), Providence.

[9] V. K. Kharchenko, Differential identities of prime rings, Algebra Logika, 17, No. 2, (1978), 220-238.

[10] M. Lorenz and D. S. Passman, Observations on crossed products and fixed rings, Comm. Algebra, 8 (1980), 743-779.

[11] W. S. Martindale, III Prime rings satisfying a generalized polynomial identity, J. Algebra, 12 (1969), 576-584.

[12] S. Montgomery and M. K. Smith, Algebras with a separable subalgebra whose centralizer satisfies a polynomial identity, Comm. Algebra, 3(2), (1975), 151-168.

[13] S. Montgomery, Fixed rings of finite automorphism groups of associative rings, Lecture Notes in Math., Vol. 818, Springer-Verlag, Berlin and N. Y. (1980).

[14] D. S. Passman, Fixed rings and integrality, J. Algebra, 68 (1981), 510-519.

[15] C_ Computing the symmetric ring of quotients, (to appear).

[16] G. K. Pedersen, $C^{*}$-Algebras and Their Automorphism Groups, LMS Monograph No. 14, Academic Press, London, (1979).

[17] M. A. Rieffel, Actions of finite groups on $C^{*}$-algebras, Math. Scand., 47 (1980), 157-176.

[18] M. E. Sweedler, Cohomology of algebras over Hopf algebras Amer. Math. Soc. Trans. 127 (1968), 205-239.

[19] Hopf Algebras, Benjamin, N. Y. (1969).

Received May 2, 1985. This work was done while the author was visiting the Mathematics Department of UCLA during 1984-85. She wishes to thank the department for its warm hospitality.

\section{BEN GURION UNIVERSITY}

BEER SHEVA, ISRAEL 


\section{PACIFIC JOURNAL OF MATHEMATICS EDITORS}

\author{
V. S. VARADARAJAN \\ (Managing Editor) \\ University of California \\ Los Angeles, CA 90024 \\ HERBERT ClEMENS \\ University of Utah \\ Salt Lake City, UT 84112 \\ R. FINN \\ Stanford University \\ Stanford, CA 94305
}

\author{
HERMANN FLASCHKA \\ University of Arizona \\ Tucson, AZ 85721 \\ RAMESH A. GANGOLLI \\ University of Washington \\ Seattle, WA 98195 \\ VAUghan F. R. JONES \\ University of California \\ Berkeley, CA 94720 \\ ROBION KIRBY \\ University of California \\ Berkeley, CA 94720
}

C. C. MOORE

University of California Berkeley, CA 94720

H. SAMELSON

Stanford University Stanford, CA 94305

HAROLD STARK

University of California, San Diego La Jolla, CA 92093

\section{ASSOCIATE EDITORS}
R. ARENS
E. F. BECKENBACH
B. H. NEUMANN
F. WOLF
K. YOSHIDA (1906-1982)

\section{SUPPORTING INSTITUTIONS}

UNIVERSITY OF ARIZONA

UNIVERSITY OF BRITISH COLUMBIA

CALIFORNIA INSTITUTE OF TECHNOLOGY

UNIVERSITY OF CALIFORNIA

MONTANA STATE UNIVERSITY

UNIVERSITY OF NEVADA, RENO

NEW MEXICO STATE UNIVERSITY

OREGON STATE UNIVERSITY
UNIVERSITY OF OREGON UNIVERSITY OF SOUTHERN CALIFORNIA

STANFORD UNIVERSITY

UNIVERSITY OF HAWAII

UNIVERSITY OF TOKYO

UNIVERSITY OF UTAH

WASHINGTON STATE UNIVERSITY

UNIVERSITY OF WASHINGTON 


\section{Pacific Journal of Mathematics}

Vol. 125, No. 1 September, 1986

Gilles Christol, Fonctions et éléments algébriques $\ldots \ldots \ldots \ldots \ldots \ldots \ldots \ldots$

Jo-Ann Deborah Cohen, Extensions of valuation and absolute valued

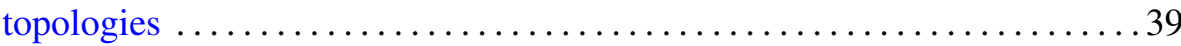

Miriam Cohen, Smash products, inner actions and quotient rings . . . . . . 45

Mikio Furushima, On the singular $K-3$ surfaces with hypersurface

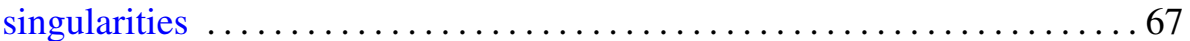

Gerhard Gierz and Boris Shekhtman, A duality principle for rational

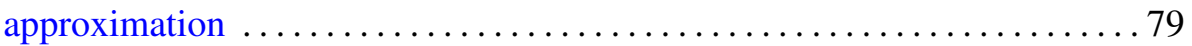

Anthony Wood Hager, A description of HSP-like classes, and

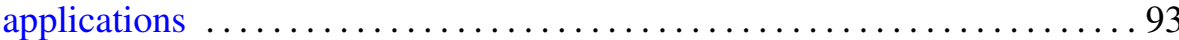

George Alan Jennings, Lines having high contact with a projective

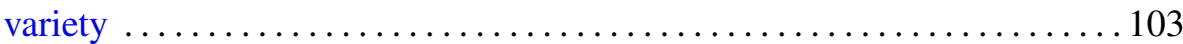

John Lott, Eigenvalue bounds for the Dirac operator . . . . . . . . . . . 117

Denis Laurent Luminet, A functional calculus for Banach PI-algebras . . . . 127

Shizuo Miyajima and Noboru Okazawa, Generators of positive

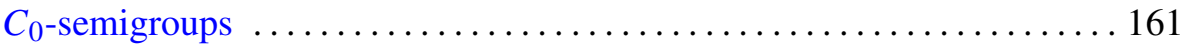

Takemi Mizokami, On functions and stratifiable $\mu$-spaces $\ldots \ldots \ldots \ldots \ldots 177$

Jeff Parker, 4-dimensional $G$-manifolds with 3-dimensional orbits . . . . . 187

Elias Saab and Paulette Saab, On Peł czyński's properties (V) and (V*) . . 205

Elmar Schrohe, The symbols of an algebra of pseudodifferential operators

Aart van Harten and Els Vader-Burger, Approximate Green functions as a tool to prove correctness of a formal approximation in a model of competing and diffusing species

Stephen Watson, Using prediction principles to construct ordered

continua 